\title{
Symmetry, beauty and belief in high-energy physics
}

\section{T} his paper engages with the aesthetics of knowledge, both in its sense as the connection between knowledge and 'aesthetic' judgements of beauty, or ugliness, and of the many 'aesthetic' - that is to say sensually perceivable - dimensions of knowledge, which are always to be seen to be constituting an epistemic factor in its production and consumption. On the one hand I analyse how in recent decades the connection between beauty and truth has been systematically employed to both inspire and guide research in high-energy physics; at the same time I also show how this use of aesthetic judgement only reveals its constitutive role in physics research when paying attention to the broad range of aesthetic strategies employed for expressing scientific knowledge.

IN PARTICULAR, an analysis of the verbal component of scientific media strategies has brought to light its entanglement with the aesthetics of religious knowledge. This aesthetic-epistemic connection plays a role in constructing and supporting the world-view shared by high-energy physicists in recent decades. I analyse an argument linking symmetry, beauty and truth which is currently being presented in a number of forms in popular and academic science writing. Its core message is that nature is characterized by one or more 'beautiful' symmetries which are 'hidden' or 'spontaneously broken', and as such do not manifest themselves in observable phenomena. Talented and committed theorists may discover such hidden symmetries and the mechanism of their breakdown by physical-mathematical research, guided by an aesthetic sense which helps them to find their way beyond the apparent asymmetry of phenomena. Experimenters should rather aim at gaining access to new manifestations of hidden symmetries by developing methods of exploring phenomena at higher energies. The existence of hidden, spontaneously broken symmetries in nature and the human ability to unveil them stand as hypothetical, yet unquestioned assumptions at the core of this world-view, and belief in them is fostered by a strategy of naturalization which relies heavily on religious themes and imagery.

\section{Introduction}

Since the early twentieth century theoretical constructs in fundamental physics have reached such a level of formal and conceptual complexity that, despite ample experimental support for their predictions, it still remains unclear how far they may be regarded as representing aspects of nature. However, physicists today express the conviction that their theories say something about the inner principles of nature well beyond their experimentally testable predictions, and often support opinions of this kind by claiming that 'beautiful' or 'elegant' theories should be regarded as more probable candidates for representing nature.

A notion which prominently features in these discourses is 'symmetry', a term which in modern mathematics indicates the invariance of a structure under a given mathematical transformation, such as a rotation in space or the exchange between left and right, but which in today's scientific writing is also used to span a broader range of practices and aesthetic experiences. Physicists claim that 'symmetries' are beautiful, and that therefore symmetric theories have a better chance of reflecting inner principles of nature, even when the theories are not (yet?) sup- 
ported by experimental evidence. The increasingly widespread use of this kind of argument in physics has been the subject of some discussion (De Regt 2002, Schummer 2006). Many questions can be and have been asked in this context, including whether a general, overarching notion of 'beauty' may be defined, how a feeling for beauty and elegance may be philosophically and/or psychologically grounded, and whether or not the connection between beauty and truth can be justified. The present paper will not deal with these topics. Taking at face value the statements on symmetry, beauty and knowledge so prominent in today's fundamental physics discourse, I will analyse the argumentative structure and rhetorical strategies which are employed to express and support these views.

I will argue that the claim that symmetry is beautiful and hence true is often presented making use of religious terms and themes, and that a very important element in this constellation is the notion of 'hidden' symmetries of nature, which are not manifest to the senses, but nonetheless shape phenomena. In section 2 I show how the beauty of symmetry is employed in contemporary particle research to support theoretical constructs, and how neither theories nor symmetries should be regarded as purely mathematical notions. In section 3 I quote a number of popular science books to discuss how a hybrid notion of symmetry as an entity present, albeit hidden, in nature is constructed, while in section 4 I offer a brief historical overview on how these arguments came to play such a central role in particle research. Finally, section 5 contains some concluding remarks on the outcomes of the investigation and on its methodological significance for the study of the interplay of science and religion.

\section{Beauty and belief in today's theoretical particle physics}

High-energy experiments have until now confirmed the predictions of a theory of particle interactions formulated in the 1970s and known as the 'Standard Model', but in the high-energy community it is generally assumed that, at energies higher than those reached so far, the predictions of the Standard Model will fail and some as-yet-unspecified 'new physics' will manifest itself. Since the 1970s, speculative models of what the 'new physics' may look like have been formulated, and these models have been

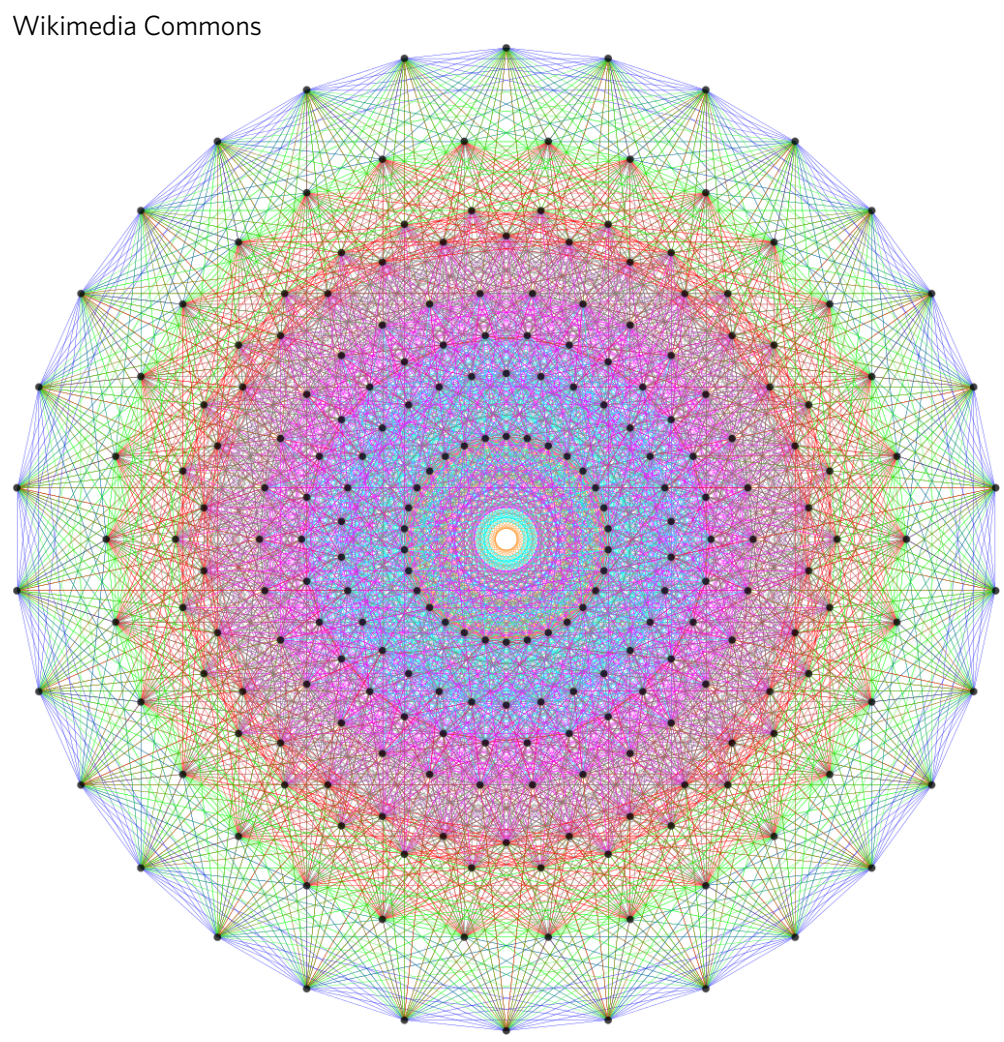

Visualisation of Lie groups, which are key structures to express 'symmetry' as mathematical invariance. The image is based on material provided by 'MyToE: a site for visualizing a Theory of Everything, dedicated to the pursuit of beauty and Truth in Nature' (theoryofeverything.org).

often supported by arguing that they are 'beautiful' or 'natural'. One of the most prominent examples is 'supersymmetry', which since the 1980 o has been touted as one of the best candidates for modelling this new physics. In 1985, Martin Sohnius began his overview 'Introducing supersymmetry', published in the prestigious journal Physics Reports, with some quite interesting remarks on symmetry, beauty and scientific knowledge:

Modern science shares with both the Greek and earlier philosophies the conviction that the observed universe is founded on simple underlying principles which can be understood and elaborated through disciplined intellectual endeavour. By the Middle Ages, this conviction had, in Christian Europe, become stratified into a system of Natural Philosophy that entirely and consciously ignored the realities of the physical world and based all its insights on thought, and Faith, alone. The break with the medieval tradition occurred when the scientific revolution 
of the 16th and 17th centuries established an undisputed dominance in the exact sciences of fact over idea, of observation over conjecture, and of practicality over aesthetics. Experiment and observation were established as the ultimate judge of theory. Modern particle physics, in seeking a single unified theory of all elementary particles and their fundamental interactions, appears to be reaching the limits of this process and finds itself forced, in part and often very reluctantly, to revert for guidelines to the 'medieval' principles of symmetry and beauty.

Supersymmetric theories (the subject of this report) are highly symmetric and very beautiful. They are remarkable in that they unify fermions (matter) with bosons (the carriers of force), either in flat space (supersymmetry) or in curved space-time (supergravity). Supergravity naturally unifies the gravitational with other interactions. None of the present model theories is in any sense complete; the hurdles on the way to experimental prediction - and thus to acceptance or rejection - have not yet been cleared. What naive immediate predictions can be made seem to be in disagreement with nature. Yet this particular field of research appears to promise solution of so many outstanding problems that it has excited great enthusiasm in large parts of the theoretical physics community (and equally large scepticism in others). In a truly philosophical spirit it has even been said of the theory that it is 'so beautiful it must be true'. (Sohnius 1985: 41-2)

This passage expressed what is today a still widely shared conviction that supersymmetry, because of its 'beautiful' properties of symmetry, should be seen as a good candidate for describing so far unobserved particle phenomena at high energies. The statement that supersymmetric theories 'unify' different kinds of particles and interactions refers to the representation of different phenomena within a single mathematical structure which displays an overarching, albeit badly broken, symmetry. Only at higher energies, so the physicists claim, is supersymmetry manifest in phenomena, while at lower energies it can only be assumed to exist on the ground of its 'beauty'. The stance of the author with respect to this epistemic constellation is somehow ambiguous. Building upon a historically fictive, but culturally well-entrenched picture of an opposition between rational, empirically grounded scientific knowledge and intuitive, aesthetically sensitive, and possibly irrational 'Faith', the author at the same time questions it, if perhaps half-jokingly. Although not explicitly stated, the similarity between supersymmetry and 'medieval', faith-inspired natural philosophies is clearly the fact that physicists are attributing to nature a characteristic ('(super)symmetry') which it does not empirically display. The justification for this step is at first just based on a perception of beauty. Interestingly, symmetry is presented as a 'medieval principle', thus suggesting that the highly technical invariances of supersymmetry can be equated to a broader principle already present in pre-modern thought, which however remains unspecified. As we shall see later, connecting the invariances of today's mathematical constructs and the pleasure they may excite among scientific practitioners to more qualitative notions of sensually perceivable beauty, regularity or harmony is a rhetorical strategy widely used in today's scientific discourse about symmetry. It serves to naturalize theoretical practices, presenting them as a more refined form of a search for beauty and truth in nature. It is an aesthetic practice which arguably displays analogies to rhetorical strategies employed in religious texts, as has been recently suggested (Lincoln 2006). As explicitly stated in the passage above, this endeavour was already being perceived by scientists in the 1980 os as being on the boundary of the traditional science-religion divide. As I shall argue in more detail later, the use of religious terminology in this context both expresses and fosters the attitude of high-energy physicists towards the knowledge they are searching for in the high-energy realm: it is a knowledge they have admittedly not yet attained, but which they believe (and expect others to believe) will be attained by following a pre-defined path in which 'symmetry' and 'beauty' serve as guidelines towards truth. But what is 'symmetry' in this context? While the term today is a standard technical expression indicating mathematical invariance, it is also often used in a broader sense, as we saw in the passage above. Before pursuing this issue further, it is therefore important to discuss some fundamental questions on the relationship between 'quantitative' and 'qualitative' modes of expression in theoretical physics research.

The idea that scientific knowledge in general, and 
knowledge of fundamental physics in particular, can and should only take the form of symbolic mathematical formulas is explicitly or implicitly accepted by practically all physicists, by a large proportion of philosophers of science, as well as by the general public. Indeed, the assumption of a divide between rigorous, logical-mathematical, empirically testable scientific knowledge and all other kinds of more or less justified belief is as well entrenched in our culture as is the science-religion divide, and is also not infrequently taken up by scholars in the humanities. However, taking a closer look at the theoretical practices of physics shows that they comprise a much broader range of medial strategies than rigorously defined symbolic formulas. Words, images, diagrams, standardized descriptions of procedures, as well as mathematical symbols employed beyond a rigorously defined area of application: all of these and many more medial, aesthetic strategies are used by today's theorists to construct and communicate their reflections. As I have discussed in detail elsewhere, theories and models in today's high-energy physics are hybrid constructs in which verbal statements are as fundamental as formulas (Borrelli 2012, 2015a). Theorists are perfectly aware of this fact, but regard this situation as a temporary one, with all non-mathematical, non-rigorous constructs considered as stand-ins for some rigorous mathematical theory which will eventually be found. This is particularly true for speculative models of 'new physics' such as supersymmetry. The hybrid nature of theoretical constructs is a significant aspect of the present subject, because notions such as 'symmetry' or 'symmetry breaking' also have a hybrid character, and religious terms and themes serve to endow them with the quality of unity.

Today the term 'supersymmetry' stands for a very broad and constantly evolving landscape of more or less sketchy models sharing as a core feature a broken invariance with respect to the 'supersymmetric' transformations Sohnius referred to. Sohnius's text was written in 1985, when the idea of supersymmetry had only recently become generally accepted as a possible research guideline. At the time, although beauty was seen as the main motivation for developing models with this special symmetry, there were reasons to expect that experiments in future years would confirm the predictions of some of these models. That, however, turned out not to be the case, and in subsequent decades supersymmetric models had to be constantly adapted to reflect their lack of empirical support. However, faith in supersymmetry was not lost, partly due to a refinement of the 'beauty' argument in its favour, which took the form of what is usually referred to as the 'naturalness' argument. The notion of 'naturalness' is another hybrid construct which has come to play a key role in theoretical highenergy research in recent decades, both for supersymmetry and more generally. I have discussed this example in some detail elsewhere (Borrelli 2015C), and here I only wish to give a brief sketch to show how important these arguments are in contemporary theoretical research.

A key argument for the existence of a new physics at higher energies states that the Standard Model is 'unnatural' and 'ugly' because it fails to unify the various (broken) symmetries it contains into a single (broken) one. The ideal of 'naturalness' as an aesthetic criterion in the search for new theories has dominated high-energy physics for the last few decades, but no single definition of 'naturalness' exists. Scientists are ready to admit that it is an 'aesthetic criterion', but, while theorists usually characterize it by means of complex technical features of models with spontaneously broken symmetries, experimenters rather point out how 'unnatural' it is that particles with similar properties, such as the six quarks, have very different mass values (Borrelli 2015c). Although experimental results have so far failed to deliver evidence for a 'new physics' beyond the Standard Model, the aesthetic ideal of naturalness still remains very relevant. At the beginning of their research paper 'Unification and new particles at the LHC' (2016) prominent theorists Nima Arkani-Hamed, Raffaele Tito D’Agnolo, Matthew Low, and David Pinner state that

Direct and indirect searches for new physics at the weak scale have produced only null results so far. It is looking increasingly more likely that the widely shared and deeply rooted belief in a perfectly natural explanation for the origin of the electroweak scale is not correct, just as we have not seen a natural solution to the cosmological constant problem. On the other hand, belief in naturalness, especially in the context of low-energy supersymmetry (SUSY), was associated with a spectacular quantitative success - supersymmetric gauge coupling unification - as well as a beautiful and calculable picture for dark matter candidates. (ArkaniHamed et al. 2016: 1) 


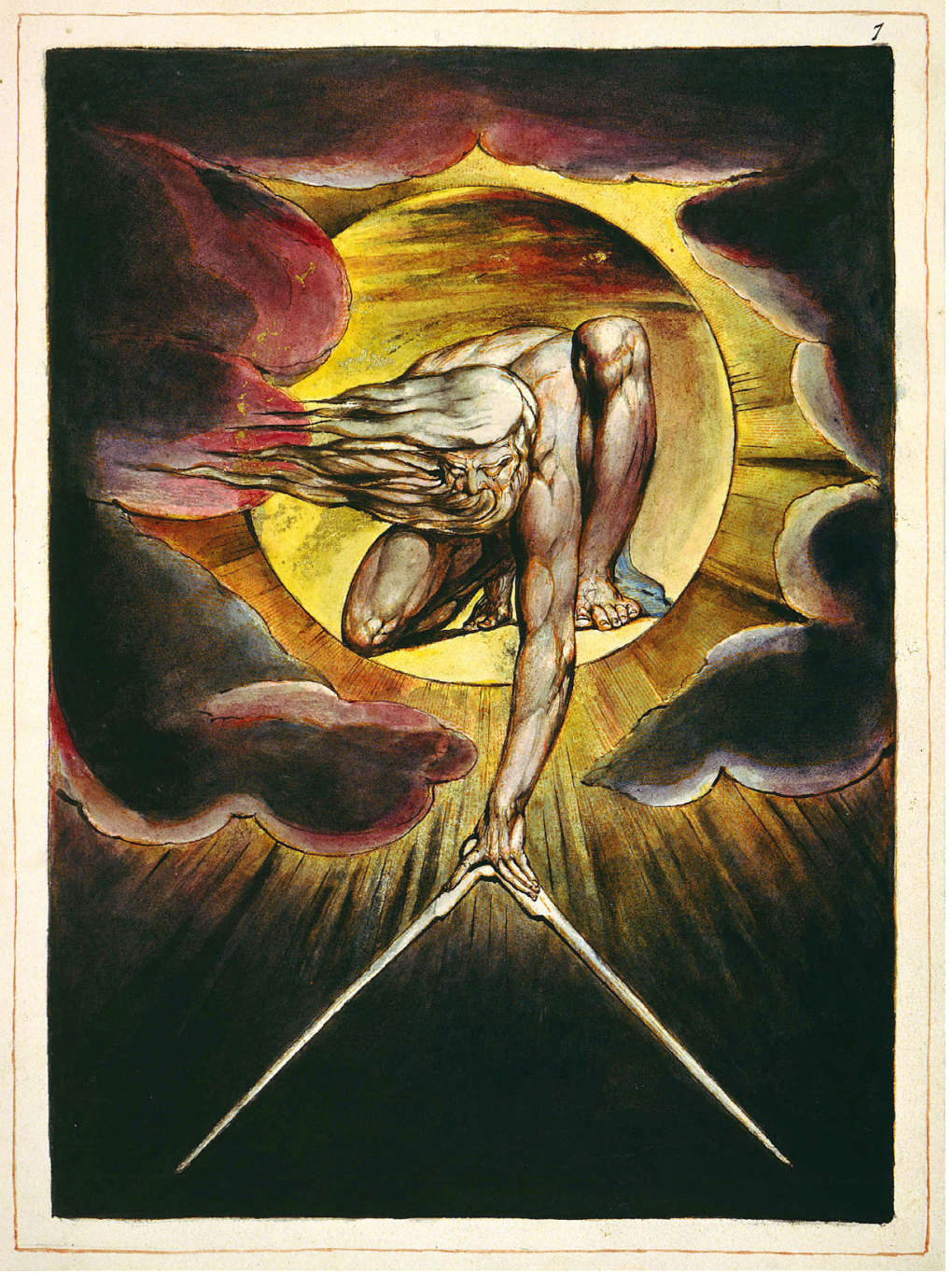

William Blake, The Ancient of Days setting a Compass to the Earth, frontispiece to copy K of Europe a Prophecy in the collection of the Fitzwilliam Museum, Cambridge University, first printed 1794. This image appears on the cover of Stewart and Golubitsky 2011.

The 'spectacular' and 'beautiful' successes of supersymmetry mentioned above are of a purely mathematical nature, but their aesthetic appeal is presented as a motivation for continuing to believe in naturalness despite the lack of empirical evidence against the allegedly unnatural Standard Model. The belief that the Standard Model is unnatural is today not only common among theorists, but is also shared by experimental physicists and plays a role in motivating their own enterprise. If the Standard Model is unnatural, so the argument goes, than the predictions of a more natural theory, such as supersymmetry, should manifest themselves at higher energies, which therefore should be experimentally explored by building new particle accelerators and detectors. Thus, although the notion of naturalness emerged in the context of theoretical research, in the course of the last few decades of the twentieth century it came to encompass and motivate a much broader range of research practices. The same can be said more in general for the beauty of symmetry, an idea which certainly developed in theoretical contexts, but today has a much broader scope. To understand better how 'symmetry' is presented as a hidden principle of nature we shall turn to more popular science writing, which is directed not only at laypersons and students, but also at members of the high-energy physics community.

\section{Beauty and symmetry in popular science writings}

Academic and popular science books presenting symmetry as a 'beautiful' inner principle of nature started appearing during the 1960s, and since then their number has been constantly increasing (De Regt 2002, Schummer 2006). Many of them have suggestive titles where symmetry, beauty and knowledge combine with religious terms: Why Beauty is Truth. The History of Symmetry (Stewart 2008), Fearful Symmetry: Is God a Geometer? (Stewart and Golubitsky 2011), Fearful Symmetry: the Search for Beauty in Modern Physics (Zee 1986), A Beautiful Question: Finding Nature's Deep Design (Wilczek 2015), Symmetry and the Beautiful Universe (Lederman and Hill 2007), The Elegant Universe: Superstrings, Hidden Dimensions, and the Quest for the Ultimate Theory (Greene 2003). These texts provide pedagogical, yet advanced presentations of the mathematical invariances which play a role in today's physics research, but at the same time they connect these symmetries with a broader range of aesthetic experiences of an artistic and religious nature. I shall argue that this presentation is extremely important to naturalize the ideas of 'symmetry', letting mathematical invariances appear as one manifestation of a universal human experience that has served as a (more or less successful) guideline in the search for knowledge about nature. As representative of many similar texts, I quote here from the first pages of Symmetry and the Beautiful Universe by the Nobel Prize winning physicists Leo Lederman and Christopher Hill: 
What is symmetry? Symmetry is ubiquitous. Symmetry has myriad incarnations in the innumerable patterns designed by nature. It is a key element, often the central or defining theme, in art, music, dance, poetry, or architecture. Symmetry permeates all of science, occupying a prominent place in chemistry, biology, physiology, and astronomy. Symmetry pervades the inner world of the structure of matter, the outer world of the cosmos, and the abstract world of mathematics itself. The basic laws of physics, the most fundamental statements we can make about nature, are founded upon symmetry. (Lederman and Hill 2007: 13)

Lederman and Hill's presentation of 'symmetry' is first and foremost a reification: symmetry is something which manifests itself in aesthetically perceivable nature, in all human activities and in the sciences, and as such can be assumed to also exist in the inner world' of matter, the 'outer world' of the cosmos and the 'abstract world' of mathematics. But what is symmetry here? The reader is at first left to imagine this for herself. The 'symmetries' of science, matter, the cosmos and mathematics, which later in the book will be characterized as mathematical invariances, are set on a par with qualitative impressions of harmony, elegance and beauty, and all of them become manifestations ('incarnations') of one and the same principle, which is however left unspecified. The important point is that 'symmetry' exists, and as such can be and should be searched for. We note the use of religious terminology ('incarnation') and pantheistic imagery: they are not simply metaphors for the use of lay readers, but rather a conscious strategy to make use of traditionally powerful patterns to reify and naturalize a general notion of 'symmetry' which will comprise, but not be exhausted by, mathematical invariance. In the following passages, the authors link the ubiquitous principle to the capability humans have of perceiving it as an innate, timeless aesthetic experience of beauty, harmony and the divine:

We first encounter symmetries in our experiences as children. We see them, we hear them, and we experience situations and events that seem to have certain symmetrical interrelationships. We see the graceful symmetry of a flower's petals, of a radiating seashell, of an egg, of a noble tree's branches and the veins of its leaves, of a snowflake, or of the line of a seashore horizon dividing the sky from the sea. ... We hear the symmetries of a drumbeat or of a simple sequence of tones in a song or in the call of a bird. ... Humans, for thousands of years, have been drawn instinctively to equate symmetry to perfection. Ancient architects incorporated symmetries into designs and constructions. Whether it was an ancient Greek temple, a geometrical tomb of a pharaoh, or a medieval cathedral, each reflected the kind of abode where a 'god' would choose to reside. Classical poetry - embodied in such masterworks as the Iliad, the Odyssey, and the Aeneid-invokes symmetrical lyric tempos to celebrate the goddesses or muses of tales and songs. A grand Bach organ fugue, echoing through the rafters of a magnificent cathedral, seems to emanate with a mathematical symmetry, as if reaching down from the vaults of heaven. Symmetry invokes mood, like the sunset on an uninterrupted ocean horizon. The symmetries that we sense and observe in the world around us affirm the notion of the existence of a perfect order and harmony underlying everything in the universe. Through symmetry we sense an apparent logic at work in the universe, external to, yet resonant with, our own minds. (Lederman and Hill 2007: 13-14)

Not only is natural and artistic beauty associated with symmetry, but so also, and indeed especially, is the religious experience. Lederman and Hill claim that humans have made use of 'symmetry' precisely in those moments when they wish to honour and adore divinities. On a superficial reading, one might think that the authors are simply pointing out the presence of some ornamental patterns in artistic and religious artefacts, or of regularities and repetitions in music or architecture, and that these elements can be unproblematically characterized as mathematical invariance, but this is not the case. As we shall see in more detail in section 4 , the notion of symmetry as mathematical invariance only emerged in the nineteenth century, and trying to project it into ornamental patterns or musical harmony is a very problematic endeavour. There is no immediate aesthetic perception of 'symmetry' as there may be of more basic, potentially religiously significant aesthetic categories, such as up/down, left/right or light/darkness. As we 
shall see later on, the notion that there is some single principle in nature which can be sensually perceived as 'beautiful' and linked to mathematical symmetries emerged hand in hand with the growing importance of invariances in physics research. However, it is an idea which was not forged just to convince experimenters or laypersons of the importance of symmetry, but rather reflected a deep belief shared by a growing number of physicists in the late decades of the twentieth century. In his Fearful Symmetry, the theoretical physicist Anthony Zee explicitly makes this issue the main topic of the volume:

In Fearful Symmetry, I wish to discuss the aesthetic motivations that animate twentieth-century physics. ... [A] esthetics has become a driving force in contemporary physics. Physicists have discovered something of wonder: Nature, at the fundamental level, is beautifully designed. ... I wish to explain how the aesthetic imperatives of contemporary physics make up a system of aesthetics that can be rigorously formulated. As my art history professors used to say, one has 'to train one's eyes'. ... [P]hysicists have to train their inner eye to see the universal principles guiding Nature's design. (Zee 1986: xii, 3-4)

Such expressions display a similarity to descriptions of a divine spirit permeating all phenomena, but which only a chosen few may come to recognize through their aesthetic sensitivity and (re)search commitment. While anyone can appreciate the 'symmetry' of flowers or music, only physicists with a trained inner eye can unveil the hidden symmetry of nature. From this point of view, much of the development of theoretical high-energy physics has been a posteriori rewritten as a search for hidden symmetry in phenomena, as for example in the article 'Symmetry: a "key to nature's secrets"' by the Nobel laureate of physics Steven Weinberg:

When I first started doing research in the late 1950s, physics seemed to me to be in a dismal state. ... Nature, like an enemy, seemed intent on concealing from us its master plan. At the same time, we did have a valuable key to nature's secrets. The laws of nature evidently obeyed certain principles of symmetry, whose consequences we could work out and compare with observation, even without a detailed theory of particles and forces. There were symmetries that dictated that certain distinct processes all go at the same rate, and that also dictated the existence of families of distinct particles that all have the same mass. Once we observed such equalities of rates and masses, we could infer the existence of a symmetry, and this we thought would give us a clearer idea of the further observations that should be made, and of the sort of underlying theories that might or might not be possible. It was like having a spy in the enemy's high command. (Weinberg 2011)

In this passage, the trope of searching for nature's secrets is mingled with military, rather than aesthetic themes, and the search for symmetry is described in terms reminiscent of pre- or early modern texts of natural magic. The references to enemies, concealment and spies serve to underscore the difficulty of the path of initiation into nature's secrets. However, they also link to a more pragmatic aspect of the historical-epistemological constellation; namely the fact that no symmetric model may immediately describe particle phenomena, since the latter are usually not symmetric. In other words, the beauty of microphysical symmetry cannot be so immediately perceived like that of flowers or music. Because of this, the rhetoric of beauty, symmetry and truth in today's fundamental physics comprises a further element: symmetry breaking. The breakdown of nature's symmetry is a necessary step enabling theories to connect to phenomena, but in the views of contemporary physicists nature's symmetry somehow remains present, albeit hidden. As Weinberg wrote in the passage quoted above, hidden symmetries 'dictate' features of observable phenomena, which in turn 'obey' them. Like a divine spirit, symmetry is not simply hidden behind phenomena, but constitutes an active principle both causally generating and formally shaping them through symmetry breaking. In Fearful Symmetry: Is God a Geometer? (1992) Ian Stewart and Martin Golubitsky write:

Fearful Symmetry isn't a theology book, or a geometry text. It's about the role of symmetry in pattern formation .... There's a ... surprise regarding symmetry: symmetric causes can have asymmetric effects ... . This paradox, that symmetry can get lost between cause and effect, 
is called symmetry-breaking. In recent years scientists and mathematicians have begun to realize that it plays a major role in the formation of patterns. (Stewart and Golubitsky 1992: xvii-xviii)

The idea that a 'hidden' or 'spontaneously broken' symmetry is at the root of all phenomena is a core item in contemporary fundamental physics. The notion of a special kind of symmetry breaking which somehow preserves symmetry is an essential complement to the view that symmetry principles rule nature. There is no unique, rigorous mathematical theory of spontaneous symmetry breaking, but only a broad range of models which are verbally interpreted in that sense (Borrelli 2015a). In this ambiguous situation, the use of religious imagery and rhetoric provides a powerful strategic tool for supporting the 'paradoxical' idea that symmetry can be lost, but still present. The paradox of spontaneous symmetry breaking expresses a tension between knowledge and non-knowledge which might at first sight seem to fit a more religious than scientific practice (Grieser 2015). Yet it is this tension that currently motivates much research. 'Symmetry' is in this context no longer merely a mathematical invariance, but rather a multi-layered conceptual framework embedding and connecting a broad range of experimental and theoretical practices of scientific research with the ideals and goals motivating them. Pursuing these activities becomes a search for clues about a hidden layer of reality. In his reflections about the role of symmetry in science, theorist Amaury Mouchet (2013) even suggests that symmetry may provide clues to the nature of rational thought:

[O]riginally being an aesthetic criterion, symmetry evolves in science into an efficient method of classification before it becomes a powerful theoretical tool to guide and even dictate our way of constructing models and theories.... I will try to show how the modern interpretations of symmetry allow to give a precise and, above all, coherent meaning of the terms 'objective' and 'regularity'. It provides a rigorous ground for the meaning of universality. It also concerns the quantitative approach of inference and understanding. ... Last but not least, these conceptions of symmetry shed light on a (the?) characteristic of intelligence, namely modelling, and on an elementary process necessary for rational thinking, namely abstraction.

(Mouchet 2013: 661-2)

According to this view, the scientists' search for nature's hidden symmetry is not only a fact-driven enterprise of knowledge production, but also - and maybe primarily - the highest expression of the key feature of human nature: rational thought. As Ledemann wrote: 'Through symmetry we sense an apparent logic at work in the universe, external to, yet resonant with, our own minds' (Lederman and Hill 2007: 14). As explicitly, if jokingly, suggested by Stewart and Golubitsky, today's physicists seem to propose an updated version of the God-geometer argument featuring the mathematical theory of symmetries instead of a pair of compasses. In both cases the argument is the same: when finding expressions of mathematical structures in nature, the human mind is making use of its imperfect, yet in some sense divine rational faculty. The knowledge attained in this way is necessarily true, and at the same time proves the presence of a divine component in Man. Through the beauty of symmetry, the God-geometer argument, which is based on rationality, is connected to an aesthetic one, so that a bridge is established between the theorists' intuition of 'mathematical beauty' and the more general perceptions of beauty, elegance, and harmony also shared by experimental physicists and the general public. Moreover, this epistemic and aesthetic constellation both supports and is supported by the image of the theoretical physicist as a 'genius' which has come to dominate scientific and popular culture in the later twentieth century, and is epitomized by Albert Einstein (Barrow 2008: $433-7)$.

\section{Symmetry, beauty and truth in high-energy physics: a historical perspective}

Despite often-repeated claims that an aesthetic sensibility for mathematical invariances is inbuilt in human nature, historical studies have shown that the notion of invariance of a system under a mathematically defined transformation only emerged near the end of the eighteenth century and that it is anachronistic to interpret earlier works as 'implicit' applications of the modern symmetry concept (Hon and Goldstein 2008: 27-48; Schummer 2006). Historians and philosophers Giora Hon and Bernard 
Goldstein have reconstructed the way in which the term 'symmetry' was initially connected to a qualitative notion of harmony between the parts and the whole, and as such had an aesthetic value which only later came to be associated with the idea of mathematical invariance. Notions of mathematical invariance were spelled out and developed only during the nineteenth century, in the context of both mathematical and physical research, and until the 1950 symmetries were employed to study physical phenomena only by mathematicians interested in physics and by a small number of mathematically-minded physicists (Borrelli 2015b).

Symmetries only became a mainstream tool of physics research around the middle of the 1950s, when a development took place whose importance for the history of symmetry in physics can hardly be overestimated: theorists Chen Ning Yang and Tsung Dao Lee proposed that certain phenomena should be interpreted as evidence of a breakdown of the invariance of particle interactions with respect to the exchange of left and right, that is to say, a loss of mirror symmetry (Franklin 1979). Up to that time, mirror symmetry had been regarded by scientists as a fundamental property of nature, like rotational or translational invariance, but soon Yang and Lee's hypothesis was experimentally confirmed and theorists started developing more or less sketchy ideas of how a model with broken mirror symmetry might look like. A particularly important proposal was put forward by Julian Schwinger, who suggested that particle interactions are characterized by a 'hierarchy of symmetries' in which a fundamental higher symmetry, through an as-yet-unclear mathematical effect is effectively lowered so, as to give rise to observed non-symmetric phenomena, such as the violation of mirror symmetry (Schwinger 1957). Ideas similar to those brought forward by Schwinger in the USA were proposed more or less at the same time in Europe by Werner Heisenberg, who suggested that while the fundamental equations of particle interactions were symmetric, for some reason only their asymmetric solutions were actually realized in nature (Heisenberg 1957). In the course of the 1960s the combination of both views served as a guideline for many other authors in developing what soon became known as 'spontaneous symmetry breaking. I have analysed elsewhere the emergence of spontaneous symmetry breaking, noting how it was - and still is - not a rigorous mathematical construct, but rather a hybrid construction comprising both mathematical and verbal elements (Borrelli 2015a).

The idea of modelling particle phenomena in terms of a combination between symmetry and symmetry breaking rapidly became a driving heuristic principle in theoretical research, also spurred by more or less robust empirical support from experiments at increasingly high energy. From 1960 onwards the use of invariance principles in theoretical high-energy physics became mainstream, and a broad landscape of symmetries emerged, which different groups of theorists regarded as possible candidates for modelling particle phenomena. Among them was one particular kind of symmetry ('local gauge symmetry') which, in the course of the 1970s, established itself as the empirically most successful option through a series of interconnected theoretical and experimental developments. The resulting theory of particle phenomena was the Standard Model, which was - and still is - based on a combination of local gauge symmetry and its spontaneous symmetry breakdown. As we saw in section 2, the Standard Model is today widely regarded as a non-ultimate theory because it fails to unify all symmetries in a single broken scheme. Interestingly, this idea emerged in the 1970s, when experimental support for Standard Model predictions was still pouring in. At the same time, the first speculative models of particle interactions were proposed as 'more natural' alternatives to the Standard Model, among them Great Unified Theories, supersymmetry and string theory. At this stage, as we saw, the use of the 'beauty is truth' argument was widely accepted in the scientific community, but when did this trend start? Or, more precisely: when did mathematical invariances start being considered as 'beautiful', and since when was their beauty employed as evidence that symmetric theories represented hidden aspects of reality even beyond their experimentally successful predictions?

Until the 1950 symmetries were employed in the study of nature only by a relatively small group of persons, mainly mathematicians, and symmetries and their beauty were not an issue in the physics community. As for popular monographs on symmetry, only a handful appeared before the middle of the twentieth century, and the most prominent among them was probably Francis Mauritius Jaeger's Lectures on the Principle of Symmetry (1917). Jaeger's book was aimed at making students of mathematics and natural philosophy aware of 'a principle of which 


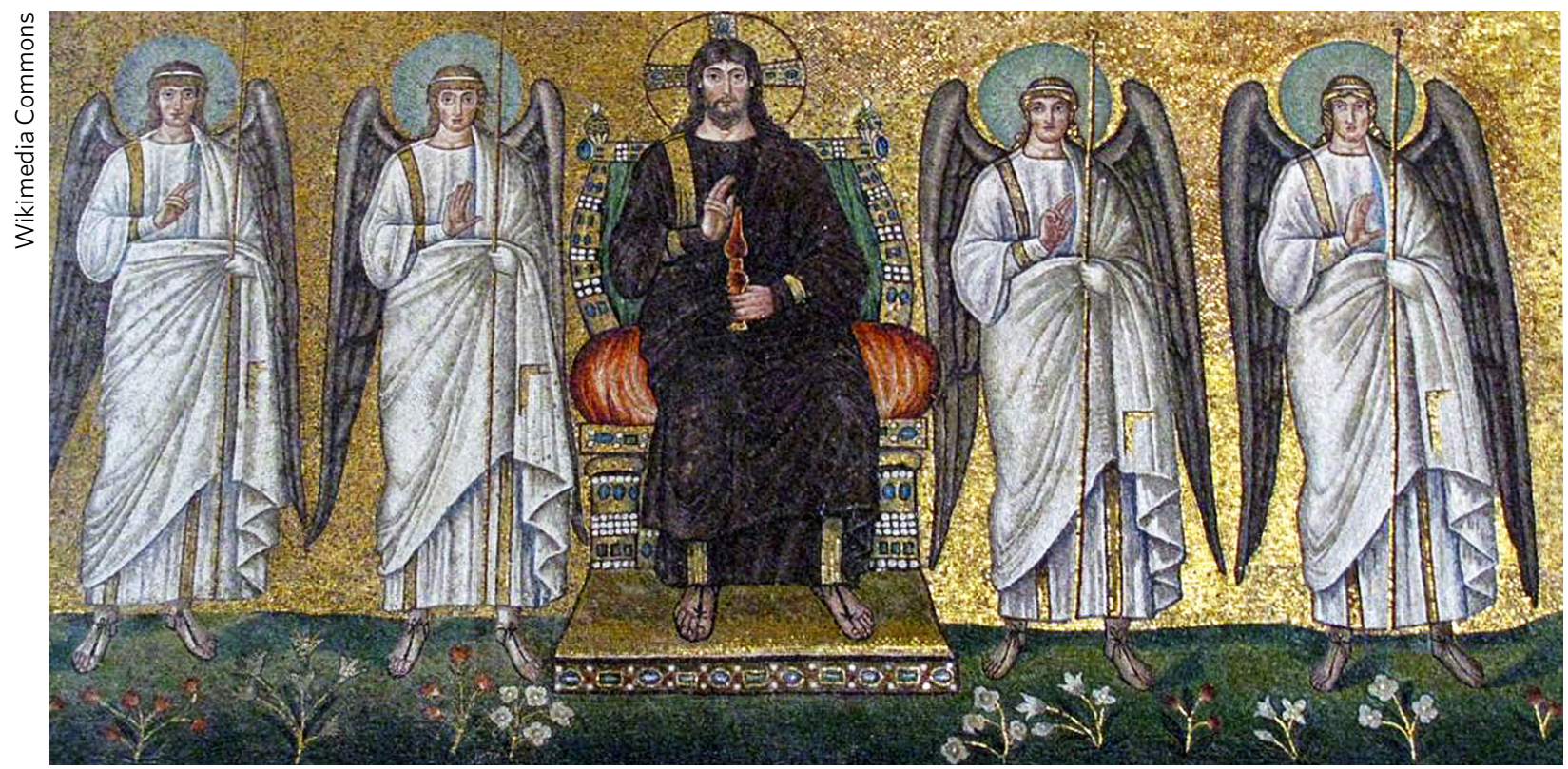

Christ surrounded by Angels and Saints, mosaic from the Chruch of S. Apollinare Nuovo (Ravenna, Italy), early 6th century. This image was shown by Hermann Weyl as an example of a slight break of left-right symmetry in art (Weyl 1952: 15).

the significance in the morphological description of objects, as well as in the definition of chemical and physical phenomena is gradually becoming more and more evident in every domain of research' (Jaeger 1917: 4). In the first chapter, he discussed the 'aesthetic value' of symmetry, explaining why symmetrical objects were perceived as aesthetically pleasing, but without ever arguing that mathematical invariances are in themselves beautiful. The rest of the book was devoted to the use of invariances in sciences, and the connection between symmetry, beauty and truth played no role here.

The first publication in which that connection took centre stage appeared in 1952, and which is still today regarded as a classic on the topic, is Hermann Weyl's Symmetry (1952), a collection of four lectures given at Princeton in 1951. Weyl was a mathematician who had contributed significantly to the development of general relativity, and his lectures expressed an aesthetic perception of mathematical beauty which was not uncommon among the mathematicians of his time. Weyl, however, explicitly linked the beauty of mathematical symmetry to artistic intuition and to the aesthetic perceptions of daily life, as would become standard later on. The book featured many pictures, and in the first three sections images from the physical sciences and mathematics were presented in combination with representations of artworks, ornaments, and living beings, establishing a bridge between physical-mathematical, biological and artistic aesthetics. Weyl began the volume by introducing two definitions of 'symmetry': harmony of the parts and whole, and left-right symmetry. He then went on to connect and generalize the two concepts to a broader mathematical notion of symmetry that had allegedly always attracted the praise of artists and scholars. Referring to Pythagoras' and Aristotle's appreciation of rotational symmetry, Weyl stated: 'It is in this tradition that a modern poet addressed the Divine Being as 'Thou great symmetry' and quoted a poem by Anne Wickham (Weyl 1952: 5; Wickham 1921). He then went on to present a series of ancient and modern works of visual art and architecture which displayed left-right symmetry, noting that 'occidental art, like life itself, is inclined to mitigate, to loosen, to modify even break strict symmetry. But seldom is asymmetry merely the absence of symmetry. Even in asymmetric design one feels symmetry as the norm from which one deviates' (Weyl 1952: 13). The perfect left-right symmetry was for Weyl a distinguishing feature of non-living physical systems, while living beings rather displayed leftright asymmetries, as shown by biological studies. Writing in 1952, Weyl was obviously not aware that physical systems, too, violate left-right symmetry and, since he died in 1955, his text was never revised to account for this discovery. Nonetheless, from the 1960 s onward the book gained a huge readership among physicists who were well aware that his views on left-right symmetry were outdated, and it was 


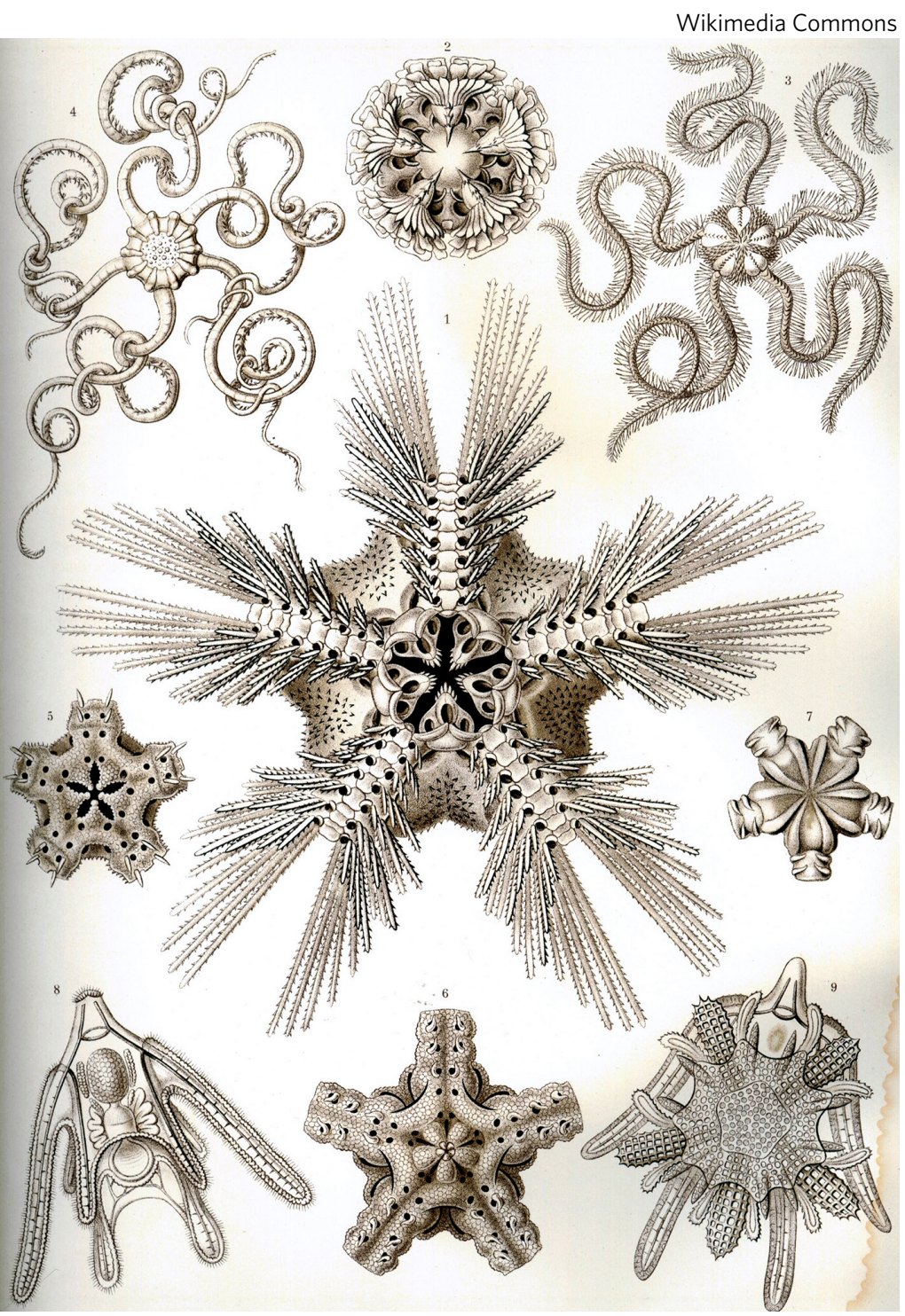

Drawings of Ophiodea (brittle stars), a kind of marine animal usually displaying radial symmetry, from Ernst Haeckel's Kunstformen der Natur (1904), plate 10. This image was shown by Hermann Weyl as an example of five-fold symmetry in living nature (Weyl 1952: 59). Images from Haeckel's volume were also employed by Chen Ning Yang (1965: 3) and are today often featured in popular texts on symmetry in nature.

reprinted many times (Weyl 2nd edn 1960, 3rd 1962, 4 th 1965,5 th 1966,6 th 1969,7 th 1973).

We may recognize in Weyl's writing some themes very popular in today's discourse on symmetry. However, he never claimed that a theory should be regarded as (more) true just because it displays symmetry. In fact, Weyl's argument is rather the reverse: in the final section of the book the empirical success of general relativity and quantum mechanics is employed to support the thesis that certain kinds of symmetries are heuristically fruitful, and potentially represent the inner order of nature. Weyl's discussion of symmetry remained isolated for a few years, and it was only around 1960 that the idea of symmetry as a beautiful principle of natural design came to be expressed increasingly often. As we saw in the previous section, that was also the period in which, after the discovery that left-right symmetry is broken in nature, the study of mathematical invariances and their (spontaneous) breakdown rapidly became a central part of mainstream particle physics research.

One of the earliest discussions of the beauty and heuristic power of symmetries after Weyl's book is found in the lecture Chen Ning Yang gave when he was awarded the Nobel Prize for the discovery of the violation of left-right symmetry (Yang 1958). Yang quoted Weyl's book, presenting 'symmetry' as a notion deeply rooted in human thought and connected both to aesthetic perception and to the quest for knowledge about nature. He began by stating: 'The existence of symmetry laws is in full accordance with our daily experience. The simplest of these symmetries, the isotropy and homogeneity of space, are concepts that date back to the early history of human thought' (ibid. 393). He then discussed the mathematical invariances of physics laws from classical mechanics to relativity and quantum theory. As is done today, Yang drew a line from everyday experiences to complex mathematical structures and characterized them all as 'simple' or 'sophisticated' symmetries (ibid. 393-4). He underscored the beauty of symmetries which are by no means apparent from daily experience' and presented it as a sign allowing scientists to hope that 'nature possesses an order that one may aspire to comprehend' (ibid. 394). Later on, the aesthetic experience of symmetry was presented as inspiring respect as if in front of some divine power:

When one pauses to consider the elegance and the beautiful perfection of the mathematical reasoning involved [in symmetry] and contrast it with the complex and far-reaching physical consequences, a deep sense of respect for the power of the symmetry laws never fails to develop. (Yang 1958: 394-5)

However, Yang did not claim that symmetrical theories are more likely to be true than non-symmetrical ones, but rather used historical developments in the physical sciences to argue that symmetries are 
useful in research and that the symmetries of empirically successful theories may be understood as representing natural principles. Thus, the religious themes in his writings cannot be understood as a means for reifying and naturalizing symmetries, but rather as a suggestion that scientific practice can be seen to be embedded in broader intellectual enterprises. A few years later, Yang reprised the themes of his Nobel lecture in his Brookhaven lecture Symmetry Principles in Physics (1965), where he quoted Weyl's statement on asymmetry in art and reinterpreted it to connect with the theme of symmetry-breaking and hidden symmetries, which had in the meantime become important in physics:

[L]et me mention the fact, especially emphasized by Hermann Weyl, that oftentimes in art one does not adhere to a strict symmetry. In fact, the existence of a general balance created by symmetrical forms, with minor perturbations causing asymmetry, may create a highly artistic effect. ... It seems that God in creating the physical world also chose to have certain symmetries largely but not perfectly obeyed. The story of the unfolding of these imperfections in the symmetries has provided much excitement in elementary particle physics in the last 10 years. (Yang 1965: 5)

Weyl had presented perfect symmetry as characteristic of physics (and possibly of the divine), and asymmetry as a feature of art and the living world. Yang, writing in a changed scientific environment, raised asymmetry to the rank of divine design principle. Once again, though, no claim was made that symmetry (or slight asymmetry) alone would be ground enough to endorse a physical theory. Such ideas were not mainstream in the 1960s, although they were occasionally employed, for example to support the hypothesis that local gauge symmetries and spontaneous symmetry breaking might provide a fruitful road to model particle interactions (Borrelli 2017). However, there can be little doubt that the increased importance of symmetries and symmetry breaking in physics had led at least some theorists to regard these methods as not only heuristically fruitful, but possibly also physically significant. It is interesting to note that, as shown by the historian Hallam Stevens, US American high-energy physicists in the 1960 s started using the quest for symmetry and unity
Wikimedia Commons

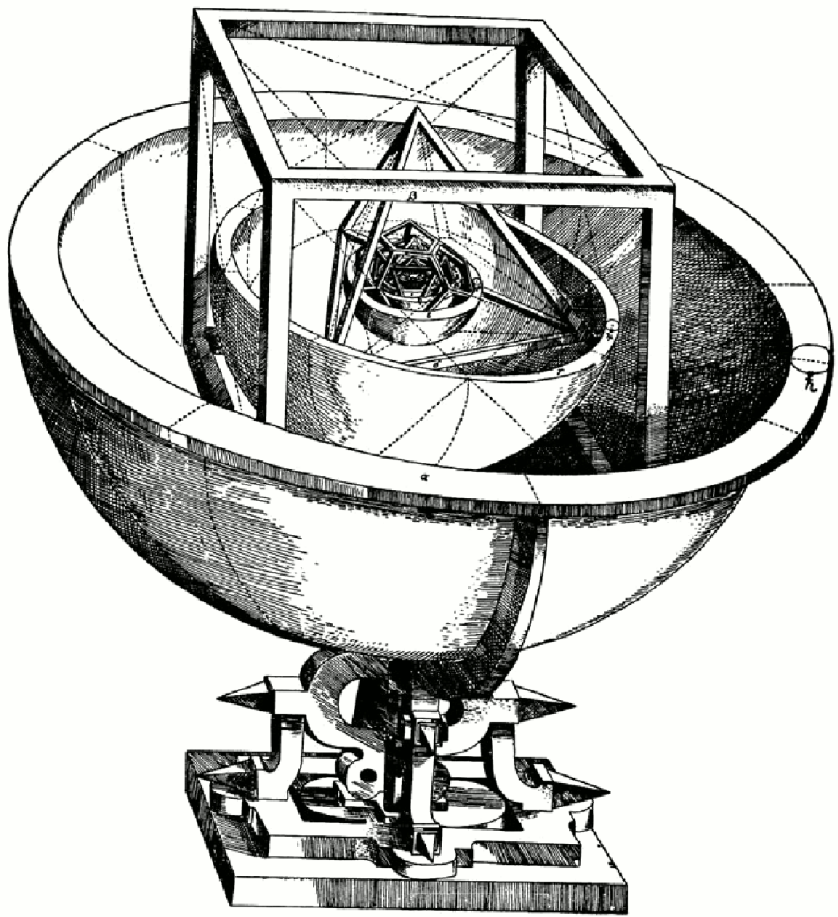

The Platonic solids as the ordering principle of the Solar System, Johannes Kepler, Mysterium Cosmographicum (1596). This image was used by Hermann Weyl to argue that Kepler 'believed he had penetrated deeply into the secrets of the Creator', thanks to symmetry (Weyl 1952: 76). This image is used as an iconic expression of the conviction that symmetry, beauty and truth belong together.

in nature in their requests for state funding, instead of arguing that fundamental research would help achieve militarily significant aims, as they had done in the 1940 os and 50 (Stevens 2003). Stevens discusses the socio-political context in which the symmetrybeauty-truth constellation was used by physicists to free fundamental research from the expectation that it would contribute to commercial and military aims, and a focus on religious aesthetics adds a very interesting perspective to this issue. This topic cannot be pursued in this article, but deserves to be analysed more in depth: it appears plausible that in this period a number of factors resonated to support physicists' belief that hidden symmetries in nature exist and can be unveiled by a theoretical research guided by aesthetic considerations and an experimental research focussed on exploring increasingly higher energies. However, it was only in the 1970 s and early 1980 s, when the first theories of 'new physics' were formulated, that the beauty of symmetry and spontaneous symmetry breaking came to be systematically used as an argument in favour of speculative theoretical 
constructs. To quote an early and very prominent example, in their seminal paper on the 'Unity of all elementary particle forces', one of the founding texts of Grand Unified Theories, Howard Georgi and Sheldon Glashow stated:

We presented a series of hypotheses and speculations leading inescapably to the conclusion that $\mathrm{SU}(5)$ is the gauge group [i.e. the symmetry] of the world - that all elementary particle forces ... are different manifestations of the same fundamental interaction .... Our hypotheses may be wrong and our speculations idle, but the uniqueness and simplicity of our scheme are reasons enough that it be taken seriously. (Georgi and Glashow 1974: 438)

Between the 1970 and early 1980 os Grand Unified Theories dominated the theoretical scene, but during the 1980 s they were replaced by other models of new physics, among them supersymmetry. In this period, the notion of naturalness emerged, to become a fundamental guideline for high-energy physics research. In the last two decades of the twentieth century, the process of reification and naturalization of symmetries was brought to completion, and in this development religious terms, themes and imagery played a very important role.

\section{Conclusions and outlook}

The present paper has engaged with the aesthetics of knowledge, both in the sense of its connection between knowledge and 'aesthetic' judgements of beauty or ugliness, and in that of the many 'aesthetic', that is, sensually perceivable dimensions of knowledge, which are always to be seen as a constitutive epistemic factor in its production and consumption. On the one hand I have analysed how, in recent decades, the connection between beauty and truth has been systematically employed both to motivate and to guide research in high-energy physics. At the same time I have also shown how this use of aesthetic judgement only reveals its constitutive role in physics research when paying attention to the broad range of strategies employed for expressing scientific knowledge. In particular, the analysis of the verbal component of scientific medial strategies has brought to light its entanglement with the aesthetics of religious knowledge. This aesthetic-epistemic connec- tion plays a role in constructing and supporting the world-view shared by high-energy physicists of the last decades. Beyond its specific results, the paper also demonstrates how fruitful an approach based on the aesthetics of knowledge can be for becoming aware of the various epistemic levels of scientific practice and of their manifold connections to its broader cultural and historical contexts. In particular, the approach brings to light the constitutive role which religious themes and imagery can play in the construction of scientific world-views. In this sense, the approach presented here offers a necessary and useful complement to the integration of traditional approaches in science studies which focus primarily either on the logical-analytical discussion of physical-mathematical constructs, or on the social, cultural and political factors shaping science.

The main thesis supported by the analysis performed in the previous pages is that in recent decades scientists working in fundamental physics research have increasingly often relied on aesthetic arguments, coupled with religious rhetoric, to garner support for their theoretical and experimental practices. I have analysed an argument linking symmetry, beauty and truth which is today presented in a number of variant forms in popular and academic science writing. Its core message is that nature is characterized by one or more 'beautiful' symmetries which are 'hidden' or 'spontaneously broken', and as such do not manifest themselves in observable phenomena. Talented and committed theorists may discover such hidden symmetries and the mechanism of their breakdown by means of physical-mathematical research, guided by an aesthetic sense which helps them to find their way beyond the apparent asymmetry of phenomena. Experimenters should rather aim at gaining access to new manifestations of hidden symmetries by developing methods of exploring phenomena at higher energies.

The existence of hidden, spontaneously broken symmetries in nature and the human ability to unveil them stand as hypothetical, yet unquestioned assumptions at the core of this world-view, and belief in them is fostered by a strategy of naturalization heavily relying on religious themes and imagery. The practices of today's physicists are presented as an unproblematic refinement of an inborn sense of beauty and of a cosmic rationality of which humans partake and which has always and everywhere led them to aesthetically perceive, worship and search for 
'symmetry'. In this context, the idea of a paradoxical act through which a symmetry spontaneously breaks itself to remain present, but 'hidden', is both intuitively appealing and intellectually challenging. It is presented as a necessary act of creation of disorder (but not chaos!) from order in nature. The manifold practices of high-energy physicists can all be related to this roughly sketched aesthetic-epistemic framework, which serves to motivate and give meaning to them, and provides a world-view shared by the fragmented expert communities of high-energy physics. This aesthetic-epistemic constellation combines the God-geometer theme with aesthetic arguments and pantheistic impressions. The religious terms and rhetoric in which it is expressed are a constitutive part of it, and allow it to resonate with similar messages well known in our culture from engagement with religious education, art, or literature, as well as fantasy books, movies and video games.

As the historical analysis has shown, the constellation is no straightforward extension of the traditional link between beauty and truth, as often claimed. First of all, discourses on the beauty of 'symmetry' only emerged in the second half of the twentieth century, and were at that time not used to support ongoing research, but rather to argue that the symmetries present in empirically successful theories might be an indication of real, but hidden principles of nature. It was only in the last two decades of that century that aesthetic judgement came to be used as a primary argument to support theoretical and experimental research in particle physics. In the second place, the theme of symmetry breaking was not present in earlier discourses, and I would like to suggest that the great success of aesthetic arguments in contemporary high-energy physics is linked to the effectiveness of the coupling of the notion of spontaneous symmetry breaking with religious themes. While exact, beautiful symmetries appear as abstract, static principles of the natural order, spontaneously broken ones become active powers generating phenomena while remaining hidden behind them. In the hands of researchers, the combination of symmetry and symmetry breaking becomes a powerful tool of world-making, truthseeking and self-realisation, since high-energy physicists looking for symmetry may imagine themselves as initiates tracing back the path from breakdown to exact symmetry. This constellation makes abandoning the search more difficult than a simple professional decision.
As shown by previous research (Pickering 1984), many further factors contributed to the establishment of speculative searches for a 'new physics' in the last decades of the twentieth century, among them the lack of experimental results in need of interpretation, a reduction in funding for fundamental research which prompted particle physicists and cosmologists to join forces, the interest of experimental physicists in a motivation for building machines capable of reaching ever higher energies, and of theorists for constructing an overarching unified rational for their models. However, it is my conviction that the connection of broken symmetry, beauty and truth analysed in this paper constitutes a further, independent factor shaping the development of fundamental research, and its connections both to the history of science and to the broader cultural, historical and aesthetic context deserve further exploration, both in themselves and in their possible correlations to the factors investigated by other authors, among them in particular Andrew Pickering (1984), Peter Galison (1997) and David Kaiser (2011).

\section{Acknowledgements}

The research presented here is part of the project Exploring the 'Dark Ages' of Particle Physics: Isospin, Strangeness and the Construction of Physicalmathematical Concepts in the Pre-Standard-Model era (ca 1950-1965) funded by the German Research Council (DFG) grant BO 4062/2-1.

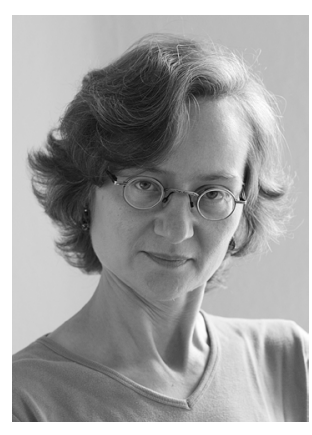

Arianna Borrelli is a historian and philosopher of natural philosophy and modern science. The focus of her research is the relationship between scientific knowledge and the strategies employed to communicate, store and transform it. Her specific fields of interest include medieval mathematical cosmology, early modern meteorology and optics, and quantum theories from their early days up to the present. She graduated in physics at Rome University and in philosophy and history at the University of Braunschweig, where she subsequently took a PhD in the history of science. She held research positions in theoretical high-energy physics and in the history and philosophy of science in Italy, Switzerland, Great Britain and Germany. She is currently working at the Technische Universität Berlin on a project funded by the German Research Council (DFG) on concept formation in early particle physics (1950-65). Her main publications include: 'From Logos to Mythos: narratives of "naturalness" in today's particle 
physics community' in Narrated Communities: Narrated Realities, H. Blume and C. Leitgeb (2015), pp. 69-83; 'Thinking with optical objects: glass spheres, lenses and refraction' in Giovan Battista Della Porta's optical writings', Journal of Early Modern Studies, 3 (2014): 38-60; Aspects of the Astrolabe: 'Architectonica Ratio' in Tenth- and Eleventh-century Europe (Franz Steiner Verlag 2008).

\section{References}

Arkani-Hamed, Nima, Raffaele Tito D'Agnolo, Matthew Low, and David Pinner, 2016. 'Unification and new particles at the LHC', Journal of High Energy Physics, 11, pp. 1-27 <doi: 10.1007/JHEP11(2016)082>

Barrow, John D., 2008. Cosmic Imagery: Key Images in the History of Science (New York, Norton)

Borrelli, Arianna, 2012. 'The case of the composite Higgs: the model as a "Rosetta stone" in contemporary highenergy physics', Studies in History and Philosophy of Science Part B, 43(3), pp.195-214 <doi: 10.1016/j. shpsb.2012.04.003>

-2015a. 'Genesis des Gottesteilchen. Narrativen der Massenerzeugung in der Teilchenphysik' in Erzählung und Geltung. Wissenschaft zwischen Autorschaft und Autorität, eds. Safia Azzouni, Stefan Böschen and Carsten Reinhardt (Weilerswist, Velbrück), pp. 63-86

-2015b. 'The making of an intrinsic property: "symmetry heuristics" in early particle physics', Studies in History and Philosophy of Science Part A, 50, pp. 59-70 < doi: 10.1016/j.shpsa.2014.09.009>

-2015c. 'From Logos to Mythos: narratives of "naturalness" in todays particle physics community' in Narrated Communities: Narrated Realities, eds. Hermann Blume, Christoph Leitgeb (Leiden, Brill), pp. 69-83

-2017. 'The Weinberg-Salam model of electroweak interactions: a genial discovery or a lucky hunch?', Annalen der Physik (forthcoming)

De Regt, Henk W., 2002. 'Beauty in physical science circa 2000', International Studies in the Philosophy of Science, 16(1), pp. 95-103 $<$ doi:10.1080/02698590120118855>

Franklin, Allan, 1979. 'The discovery and nondiscovery of parity nonconservation', Studies in History and Philosophy of Science Part A, 10(3), pp. 201-57 < doi: 10.1016/0039-3681(79)90008-6>

Galison, Peter, 1997. Image and Logic: A Material Culture of Microphysics (University of Chicago Press)

Georgi, Howard, and Sheldon Glashow, 1974. 'Unity of all elementary-particle forces', Physical Review Letters, 32(8), pp. 438-41 <doi: 10.1103/PhysRevLett.32.438>

Greene, Brian, 2003. The Elegant Universe: Superstrings, Hidden Dimensions, and the Quest for the Ultimate Theory (New York, Norton)

Grieser, Alexandra, 2015. 'Imagination des Nichtwissens. Zur Hubble Space Imagery und den Figurationen des schönen Universums zwischen Wissenschaft, Kunst und Religion' in Religion - Imagination - Äshtetik. Vorstellungs- und Sinneswelten in Religion und Kultur, eds. Lucia Traut and Annette Wilke (Bristol, Vandenhoeck \& Ruprecht), pp. 451-85
Heisenberg, Werner, 1957. 'Quantum theory of fields and elementary particles', Review of Modern Physics, 29, pp. 269-78

Hon, Giora, and Bernard Goldstein, 2008. From 'Summetria' to Symmetry: The Making of a Revolutionary Concept (Berlin, Springer)

Jaeger, Francis Mauritius, 1917. Lectures on the Principle of Symmetry (Amsterdam, Elsevier)

Kaiser, David, 2011. How the Hippies Saved Physics: Science, Counterculture, and the Quantum Revival (New York, Norton)

Lederman, Leon, and Christopher Hill, 2007. Symmetry and the Beautiful Universe (Amherst, Prometheus)

Lincoln, Bruce, 2006. 'How to read a religious text: reflections on some passages of the Chāndogya Upanisad', History of Religions, 46(2), pp. 127-39 <doi: 10.1086/511447>

Mouchet, Amaury, 2013. 'Reflections on the four facets of symmetry: how physics exemplifies rational thinking', The European Physical Journal H, 38(5), pp. 661-702 <doi: 10.1140/epjh/e2013-40018-4>

Pickering, Andrew, 1994. Constructing Quarks: A Sociological History of Particle Physics (Edinburgh University Press)

Schummer, Joachim, 2006. 'Symmetrie und Schönheit in Kunst und Wissenschaft' in Ästhetik in der Wissenschaft, ed. Wolfgang Krohn (Hamburg, Mainer), pp. $57-78$

Schwinger, Julian, 1957. 'A theory of the fundamental interaction', Annals of Physics, 2, pp. 407-34

Sohnius, Martin F., 1985. 'Introducing supersymmetry', Physics Reports, 128 (2), pp. 39-204 <doi: 10.1016/0370-1573(85)90023-7>

Stevens, Hallam, 2003. 'Fundamental physics and its justifications, 1945-1993', Historical Studies in the Physical and Biological Sciences, 34(1), pp. 151-97 <doi: 10.1525/hsps.2003.34.1.151>

Stewart, Ian, 2008. Why Beauty is Truth: The History of Symmetry (New York, Basic Books)

Stewart, I., and Martin Golubitsky, 2011. Fearful Symmetry: Is God a Geometer? (Mineola, Dover)

Weinberg, Steven, 2011. 'Symmetry: a "key to nature's secrets"', New York Review of Books, 27.10.2011 $<$ http://www.nybooks.com/articles/archives/2011/ oct $/ 27 /$ symmetry-key-natures-secrets/> (accessed 25.6.2017)

Weyl, Hermann, 1952. Symmetry (Princeton University Press)

Wickham, Anna, 1921. The Contemplative Quarry (Harcourt, Brace \& Co.)

Wilczek, Frank, 2015. A Beautiful Question: Finding Nature's Deep Design (New York, Penguin Press)

Yang, Chen Ning, 1958. 'The law of parity conservation and other symmetry laws of physics' in Les Prix Nobel en 1957 (Stockholm, Norstedt \& Söner), pp. 95-105

-1965. Symmetry Principles in Physics, Brookhaven Lecture Series, 50 (Upton, NY, Brookhaven National Lab)

Zee, Anthony, 1986. Fearful Symmetry: The Search for Beauty in Modern Physics (New York, Macmillan) 